\title{
Application of geo-statistics in calculation of a uranium deposit
}

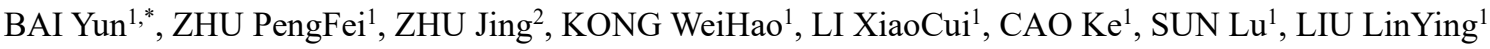 \\ ${ }^{1}$ Beijing Research Institute of Uranium Geology, Beijing 100029, China \\ ${ }^{2}$ China University of Geosciences (Beijing), Beijing 100083, China
}

\begin{abstract}
Based on the 3DMine three-dimensional mining software, a geological database model of the Changpai area is established, and a three-dimensional model of the ore body is established based on the principle and geological understanding of the circle. We carried out sample combination by a geological database model to process ore bodies of erratic high grades. We also stablished a block model, and used single assignment and ordinary Kriging method to assign value to the block model, used attribute visualization to intuitively reflect the distribution law of the ore grade in a three-dimensional space, and the block report can quickly estimate the grade, volume, ore quantity, etc., of which the results are more accurate. The common Kriging method has obvious advantages in valuation, and it is worthy for further promotion in the estimation of uranium reserves.
\end{abstract}

\section{Introduction}

Three-dimensional visualization modeling technology plays an increasingly important role in domestic and foreign mine production. It is mainly to integrate geological data from various periods and sources, set up the three-dimensional geological modeling platform, and simulate the real geological environment, so as to solve the problem of visualization and data management of internal structure and properties that cannot be solved by traditional methods, which is conducive to reserve estimation and mineralization prediction.

\section{Overview of regional geology}

The Changpai area is located in the middle of the Yangtze River uranium ore field in the south of the Zhuguang mountain rock mass, and is connected to the Mianhua pit deposit in the north. Its geological structure is in the middle of the Yangtze rift belt, clamping controlled by Niuwei mountain fault zone to the northnorth-east, Huangxi river fault zone to the north-east and oil cave fault zone to the north-west. The fault structures in this area are mainly north-north-west, north-west, and north-east groups (as shown in Figure 1). The northwest-west structural alteration belts No. 61, No. 7, No. 60 , No. 9 (southern section) are the main ore-bearing structures in this area, and there are a north-west oil cave fault and a group of north-east-facing porphyry belts. Magmatic activities are frequent in this area, which mainly composed by Yanshanian granite, the first stage and second stage Indosinian intrusional granite. Uranium mineralization in this area is strictly controlled by the fault zone, and the occurrence of the ore body is basically the same as that of the structural zone. The modeling range in this paper is $1 \mathrm{~km}$ long and $1.1 \mathrm{~km}$ wide.

1-Quaternary system; 2-Granite porphyry; 3Yanshanian fine-grained muscovite granite; 4Yanshanian fine-grained bimicite granite; 5-Yanshanian mid-fine-grained mica granite; 6-Yanshanian mediumgrained biotite granite; 7-Indosinian medium-grained small porphyry mica granite; 8-Indosinian-medium coarse-grained porphyry mica granite; 9-syenite; 10lucent porphyry; 11-structural occurrence; 12-deposit, mine pit ; 13- anomaly point; 14- structural zone and number; 15- modeling range;

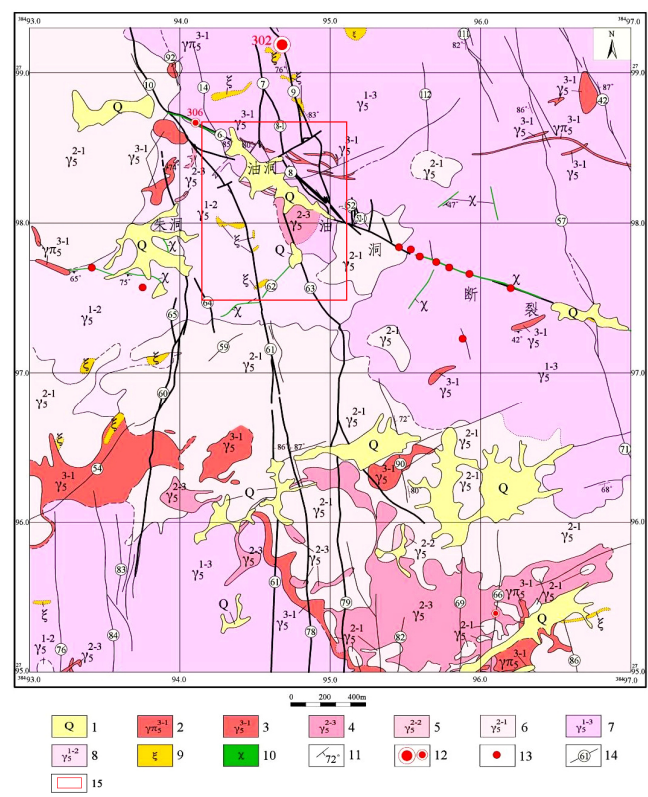

Figure 1 Geological structure of Changpai

*Corresponding author: 12303195@qq.com 


\section{Geological model construction}

This geological model construction only collected data within the modeling range, and 70 exploration line profiles and a topographic geological map with exploration lines were obtained. Based on the collected data, we built a three-dimensional geological database, and the technical route shown in Figure 2.

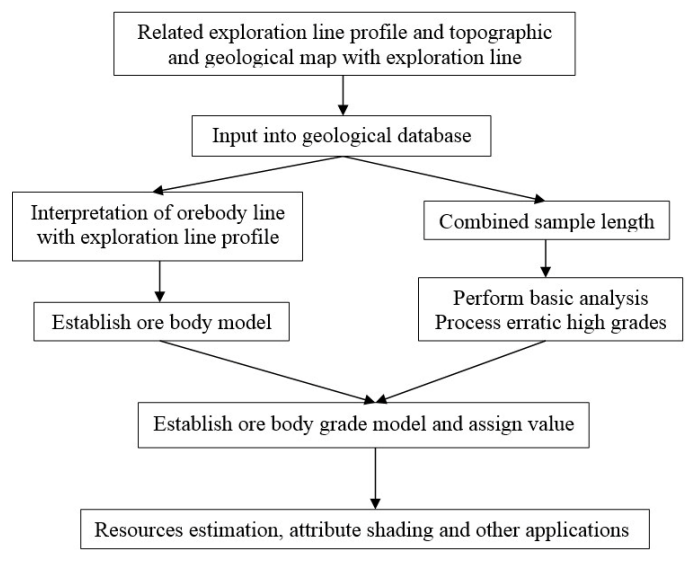

Figure 2 Model construction technology roadmap

\subsection{Geological database construction}

The geological borehole database mainly includes 4 tables, namely positioning table, inclinometer table, lithology table and grade table [1], among which grade table mainly stores the position information of grade in the borehole. All information in the database comes from the survey line profile. First, we used the planar twopoint coordinate transformation to convert the terrain geological map with exploration lines to the actual coordinates, and then the exploration line profile was converted according to the actual position of the exploration line in the plan view. Finally, based on the converted profile, we obtained the information required by the database to complete the drilling database.

\subsection{Ore body model construction}

The uranium mineralization in this area is strictly controlled by the fault zone, and the ore body is mainly produced in the north-west oil cave fault and the northwest-west structural alteration zone at No. 61, No. 7, No. 9 (southern section); The occurrence of ore bodies is basically consistent with that of structural belts. Most of the ore bodies are oriented from north-north-west to north-west, about $325^{\circ}$ to $355^{\circ}$, with a dip angle ranging from $65^{\circ}$ to $87^{\circ}$, which appear in the form of bands and lenses (as shown in Figure 3).

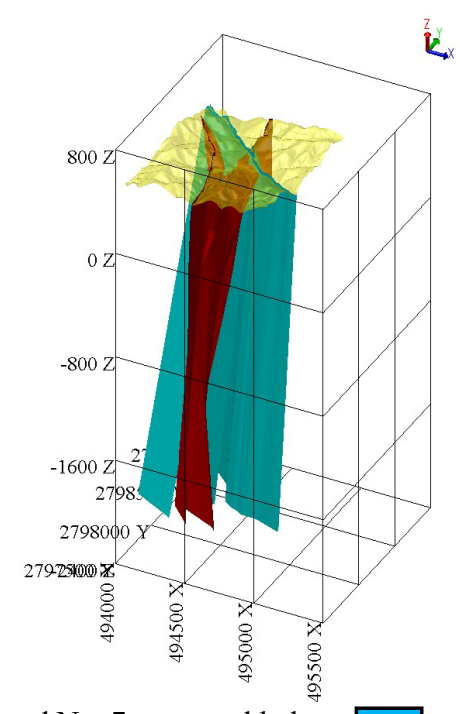

No. 61 and No. 7 structural belts, $\square$ oil cave faults, ground surface

Figure 3 Structural superposition of Changpai area

Uranium mineralization has obvious changes in the vertical direction. In the middle and upper parts, due to the frequent development of the vein body, the mineralization is discontinuous and the grade is low, while in the deeper part, because there are less veins in the later period, the ore body is of high grade. In addition, the vertical depth of mineralization is also controlled by the structure. The structure below the elevation of $-400 \mathrm{~m}$ has not been thinned out. It is speculated that there are still high-grade uranium ore bodies, and the deep part of this area still has prospecting potential [2].

According to the general industrial requirements for uranium mines specified in the "Uranium Mine Geological Prospecting Standards" (DZ/T0199-2002), and combined with the actual needs of this work, the industrial indicators adopted for the delineation of the ore body are as follows: the minimum industrial grade: $500 \times 10^{-6}$; the lowest percentage of industrial rice: 0.035 $(\mathrm{m} . \%)$; the boundary grade: $300 \times 10^{-6}$; the boundary of rice: $0.021(\mathrm{~m} \%)$; the lowest grade of ore body: $100 \times 10^{-}$ ${ }^{6}$; the percent value of the lowest enclosing ore body is $0.007(\mathrm{~m} . \%)$; the minimum recoverable thickness: 0.70 $\mathrm{m}$; the thickness of the stone removal: $0.70 \mathrm{~m}$.

The construction of the ore body model in the Changpai region combines the grade data entered in the borehole database and the ore body line extracted from the exploration line profile to interpret the ore body contour line [3], and then connects the ore body lines of adjacent sections to complete the delineation of the ore body (as shown in Figure 4). Finally, it is verified by the entity [4]. 


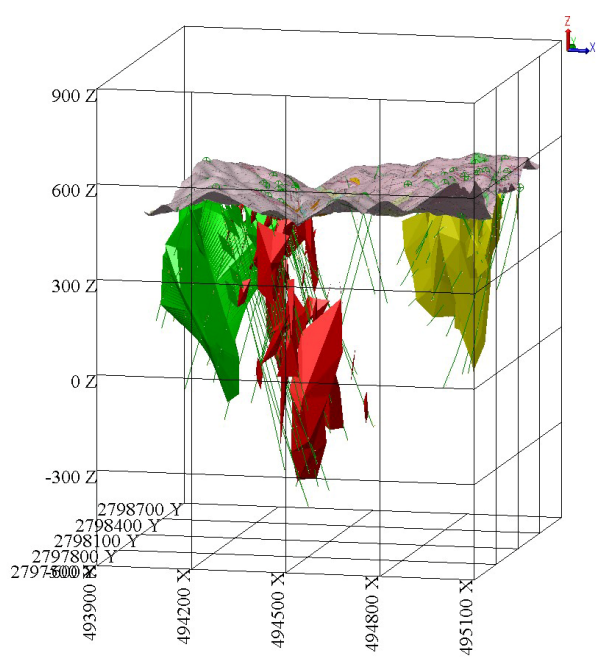

Mineralized body in zone 60

Mineralized body in zone 61 and $7 \square$ Mineralized body in zone 9 (southern section)

Figure 4 Mineralized bodies No. 61, No. 7, No. 60 and No. 9 (southern section)

\subsection{Block model construction}

We established the block model, set the unit block size, secondary module size, and used the ore body solid model as the constraint. Then we use the single assignment and ordinary Kriging method to assign the block model to prepare for future applications.

\subsubsection{Building a block model}

The factors that determine the size of the block are mainly to fully reflect the changing characteristics of the ore body. The ore body should have a large change range,

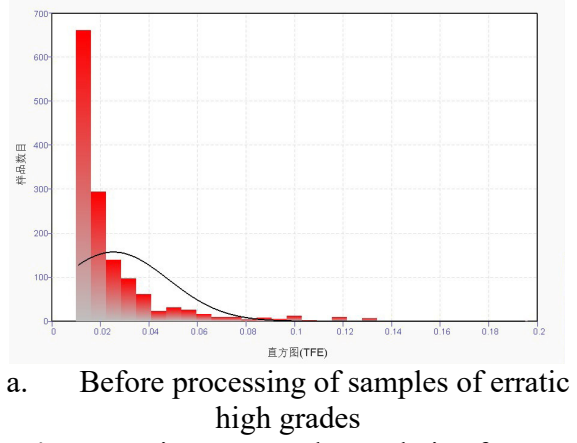

Figure 5 Histogram and cumulative frequency curve of combined sample position distribution the unit block should be small, the ore body should change slowly, and the unit block should be large. If the block size is too large or too small, the sample evaluation result will be average, and it cannot accurately reflect the characteristics of grade changes. Therefore, after determining the size of the primary unit block, the size of the secondary unit block must also be determined. The secondary unit block is generally $1 / 2$ of the primary unit block.

Considering comprehensively, the primary unit block size was set as $X: 5 \mathrm{~m}, Y: 5 \mathrm{~m}, Z: 2.5 \mathrm{~m}$, and the secondary unit block size was set as $X: 2.5 \mathrm{~m}, Y: 2.5 \mathrm{~m}$, $Z: 1.25 \mathrm{~m}$

The newly built block model is constrained by the ore body model and assigned a density value using a single assignment method. According to previous studies, the density in this area is $2.57 \mathrm{~g} / \mathrm{cm}^{3}$, which can be used for reserve estimation.

\subsubsection{Combined samples}

Using geological zone combined samples, the generated data information is stored in the attributes of spatial discrete points, and these spatial discrete points are known as sample points, which are estimated for unknown unit blocks.

Counting the discrete points in this area (as shown in Figure 5), most samples have a grade of less than 0.12 , so those with grades greater than 0.12 are labelled as samples of erratic high grades, which are generally replaced by $6 \sim 8$ times of samples of the average grade. In this study, the samples of erratic high grades were replaced by those of the 6 times average grade of 0.02 .

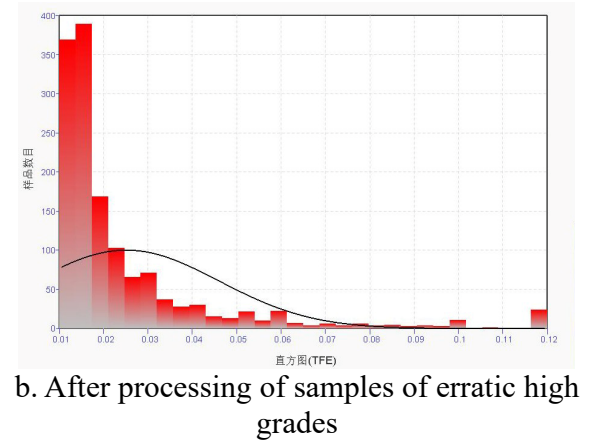

the major axis, minor axis, and short axis are consistent (as shown in Figure 6), that is, the orientation of the main axis variation function is 227 , the inclination angle is 63.18 , and the step length is 7 ; the orientation of the secondary axis variation function is 227 , the inclination angle is -26.82 , and the step length is 16 ; the orientation of the short axis variation function is 137 , and the inclination angle is 0 , the step size is 61 . The statistical characteristics are the same, and the common Kriging method can be used to assign values uniformly. 


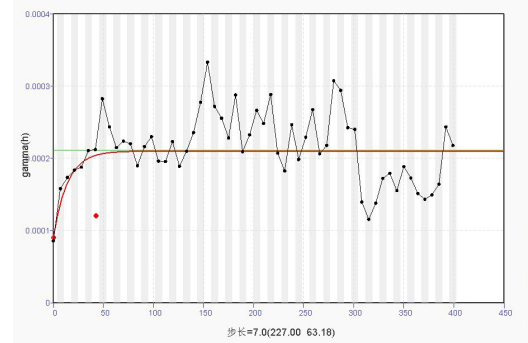

a. Spindle variation function parameters

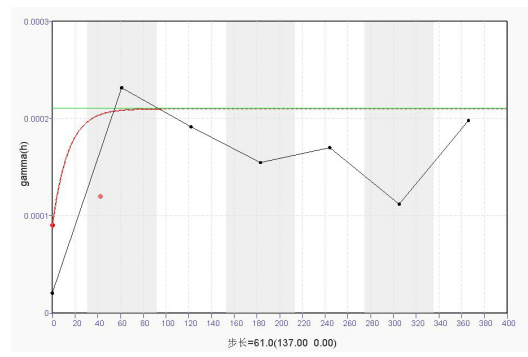

c. Short-axis variation function parameters

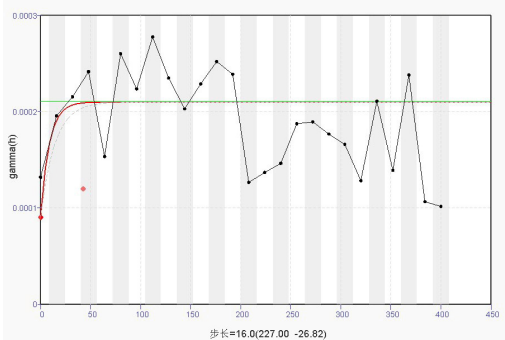

b. Axis variation function parameters

\begin{tabular}{|c|c|c|c|}
\hline Attribute & Number & Attribute & Number \\
\hline $\begin{array}{c}\text { Number of valid } \\
\text { samples }\end{array}$ & 1426 & $\begin{array}{c}\text { Standard } \\
\text { deviation }\end{array}$ & 0.01452 \\
\hline Minimum & 0.01 & $\begin{array}{c}\text { Coefficient of } \\
\text { variation }\end{array}$ & 0.67582 \\
\hline Maximum & 0.074 & Skewness & 2.13412 \\
\hline Average & 0.02148 & Kurtosis & 7.08161 \\
\hline Geometric mean & 0.01845 & $\begin{array}{c}\text { Sischel } \\
\text { coefficient }\end{array}$ & 0.25255 \\
\hline Median & 0.016 & Sisher gamma & 1.13458 \\
\hline Variance & 0.00021 & $\begin{array}{c}\text { Sischel } \\
\text { valuation }\end{array}$ & 0.02094 \\
\hline
\end{tabular}

d. Variation function analysis results

Figure 6 General Kriging estimation parameters

After fitting the parameters of the theoretical variation function, the reliability of the parameters needs to be verified. Cross-validation results show that the mean difference is close to 0 , and the variance/standard deviation approaches 1 , indicating that the fitted variable function parameters are reliable and can be used for ordinary Kriging estimation.

\section{3D geological model application}

\subsection{Reserve estimation and resource evaluation}

As an indispensable link in the geological survey work, reserves estimation has important practical significance for the development, utilization, and economic feasibility study of mines. Traditional reserves calculation methods include section method, geological block method and polygon method [5]. Calculated by traditional methods, the data to be processed is more, more cumbersome, and the efficiency is not high. Using geostatistical methods, reserves can be calculated quickly and accurately, and can be classified for processing, greatly improving work efficiency. In this study, the block model report was obtained by grade interval, mainly including grade, volume, ore quantity, etc. (as shown in Table 1).

Table 1 Report of block model reserves of uranium deposits in Changpai area Report of block model reserves

\begin{tabular}{|c|c|c|c|}
\hline \multicolumn{4}{|c|}{ Report of block model reserves } \\
\hline Grade range $(\%)$ & Volume $\left(\mathrm{m}^{3}\right)$ & Average grade $(\%)$ & Resources(Ton) \\
\hline $0.03-0.05$ & $*$ & $*$ & $*$ \\
\hline $0.05-999$ & $*$ & $*$ & $*$ \\
\hline Total & $*$ & $*$ & $*$ \\
\hline
\end{tabular}

Note: "*" refers to the volume, grade and resources of uranium ore (not to be disclosed)

The resource amount estimated by geo-statistics is $0.013 \%$ compared with the resource data provided in the deposit exploration report. The estimated result is within the allowable range of normal error, and the result is basically reliable.

\subsection{Block model attribute shading}

After the block model is assigned, each block will have its own attribute, and the block can be colored according to the different attribute values [6]. This block model has block position attributes, block length, width and height attributes, density attributes and grade attributes. According to the coloring of grade attributes, the block model is displayed to show the spatial variation of the grade of the ore body more clearly (Figure 7). There are high-grade ore bodies in the deep part of ore bodies in zones No. 61 and No. 7, and the structure has not been thinned out here. The ore bodies tend to extend deep. 


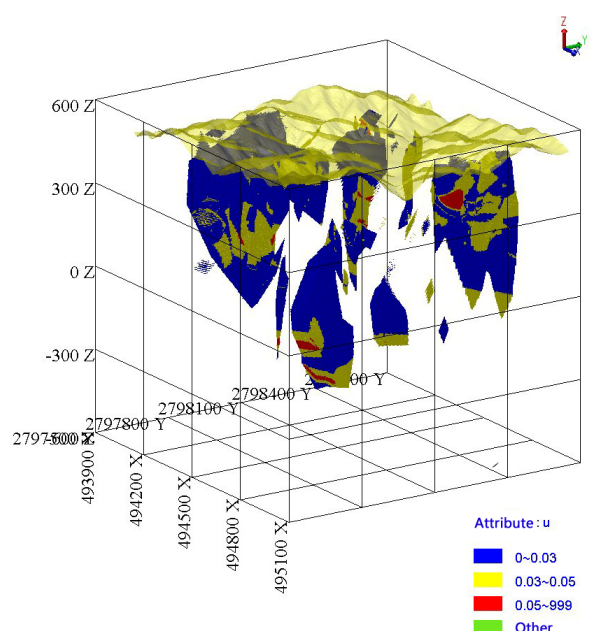

Figure 7 Color display of block model grade attributes

\section{Conclusion}

- Based on the borehole grade data and the profile of the exploration line, a three-dimensional geological model is established to visualize the shape, occurrence, and distribution of the ore body, and basically clarify the relationship between the ore body and the structure. The assigned block model demonstrates the grade distribution status and provides a basis for further prediction.

- Based on the established geological database, we performed statistics on the original grade data, identified and processed ore bodies of erratic high grades, established a block model, and assigned values to each unit block through the ordinary Kriging method, which can quickly find the average ore grade and resource quantity of different height and grade ranges. Compared with the traditional method, the error of the method in this paper is relatively small and within the acceptable range. Therefore, the method of using geo-statistics to estimate the resource reserve is worthy of promoting.

\section{References}

1. Yu Niuben, Qi Wentao, Wang Lihuan, et al. 3D geological modeling and reserve estimation based on 3DMine software. Taking a mine field in Balikun mining area of Xinjiang as an example [J]. Metal mine, 2015, 465 (3): 138-142.

2. $\mathrm{Xu}$ Wenxiong, ye Songxin. Uranium prospecting potential in the South longpai area of Zhuguang rock mass [J]. Uranium geology, 2013.29 (5): 263-267.

3. Li Xiujie, the rapid application of 3DMine software in chemical mine modeling and reserve calculation [J]. Applied chemical mineral geology, 2015, 37 (1): 35-40.

4. Zhou Deng, Jiang yongbiao. Three dimensional model construction of three Chen ore belt of Zoujiashan uranium deposit based on 3DMine [J]. Modern mining, 2016, 565 (5): 106-110.

5. Wang Jianwei, three dimensional model construction and reserve calculation based on Surpac [J]. Copper engineering, 2014, 128 (4): 60-63.

6. Zhou Deng, Jiang yongbiao. Construction and application of 3D geological model of 3DMine in No.3 ore belt of Zoujiashan uranium deposit [J]. Journal of geology, 2017, 41 (1): 93-96. 